\title{
Noise Suppression Phenomenon in Fiber Raman Amplifier
}

\author{
Georgii Felinskyi, Mykhailo Dyriv \\ Deparment of Radiophysics, Electronics, and Computer Systems, Taras Shevchenko University of Kyiv, \\ Academician Glushkov prospect, 2, 03022, Kyiv, Ukraine, e-mail: felinskyi@yahoo.com
}

\begin{abstract}
The problem of small bit error rate is actual in fiber Raman amplifiers. Our experimental data on the amplified spontaneous emission (ASE) with backward pumping as a base of optical noise evaluation are presented in this paper. Raman gain spectrum for useful Stokes low-powered signal is calculated and compared with obtained spectrum of non-coherent ASE noise. It is shown that output optical signal has better gain than the amplified spontaneous emission that leads to appreciable growth of output optical signal-to-noise ratio.
\end{abstract}

Keywords: Amplified spontaneous emission, fiber Raman amplifier, single-mode fiber, optical signal-to-noise ratio, Raman gain.

\section{INTRODUCTION}

$\mathrm{T}$ HE CREATION of light amplifiers based on stimulated Raman scattering (SRS) legally refers to the most powerful practical achievements [1] in optic telecommunication. At first, practical applications of fiber Raman amplifiers (FRA) with several pumping sources [2] high-quality amplification of optical signals with a bandwidth close to limiting for silica fibers about $13 \mathrm{THz}$ have been shown. Such amplifiers are widely applied despite the actual interdiction imposed by the theory. Really modern theory [3-5] predicts that the noise figure of any optical amplifier should be higher than the minimal quantum limit of $3 \mathrm{~dB}$. It means that the optical signal-to-noise ratio (OSNR) of the amplifier should decrease at least 2 times. The generalized quality parameter of any digital communication system is the bit error rate (BER). It depends directly on the OSNR [6]:

$$
\mathrm{BER}=\frac{1}{2} \operatorname{Erfc}\left(\sqrt{10^{\frac{\mathrm{OSNR}}{10} / 8}}\right)
$$

where $\operatorname{Erfc}(\mathrm{x})$ is the complementary error function, OSNR is in $\mathrm{dB}$ units. The noise statistics is that in the OSNR level above $12 \mathrm{~dB}$ each decibel increasing or reducing this ratio creates reduction or accordingly increases the BER at the order on value. In addition, to increase the signal transfer distance in the communication link one must not so much try to restore power losses of a signal due to attenuation in a link, but mainly the maintenance of necessary value OSNR, for example, not less than $21.6 \mathrm{~dB}$ that corresponds to $\mathrm{BER}=10^{-9}$. Hence, each optical amplifier, including FRA, theoretically should appreciably increase the BER during the digital information transfer. However, there is an obvious contradiction between theoretical performances of optical amplifier noise and real practice of their application in the optical fiber systems. In particular, standard optical communication scheme of linear signal regeneration using the sequential application of optical amplifiers becomes theoretically impossible without full signal restoration from the noise, in view of fast error accumulation with the increase in the amount of amplifiers. Even almost twenty years ago it was observed [5] that the physical phenomena, which result in extraordinary rare occurrence of mistakes in digital optical communication systems have, till now, not received unequivocal interpretation. Now the same remarks can be related to the noise performance of practical FRA. Thus, the experimental results on the amplified spontaneous emission (ASE) observation in the single-mode fiber span using the backward pumped distributed FRA are presented in this report. The calculation of the efficiency of signal Raman amplification based on on-off Raman gain for lowpowered useful signal gives us the opportunity to estimate real OSNR at fiber output.

\section{ASE NOISE MEASUREMENT AND SIGNAL GAIN SIMULATION}

We have observed ASE from the single-mode fiber span in counter direction to pump using commercial FRA unit with laser diode (LD) pump source at wavelength $1465 \mathrm{~nm}$. The experimental setup is shown in Fig.1. Output pump power from LD is directed to the $50 \mathrm{~km}$ span of standard singlemode fiber (SMF). ASE power from the fiber is registered at position B by optical spectrum analyzer (OSA). Spectral resolution of OSA was set to $1 \mathrm{~nm}$ for all ASE measurements. Input pump power levels are as follows: 100, $150,200,250$, and $300 \mathrm{~mW}$.

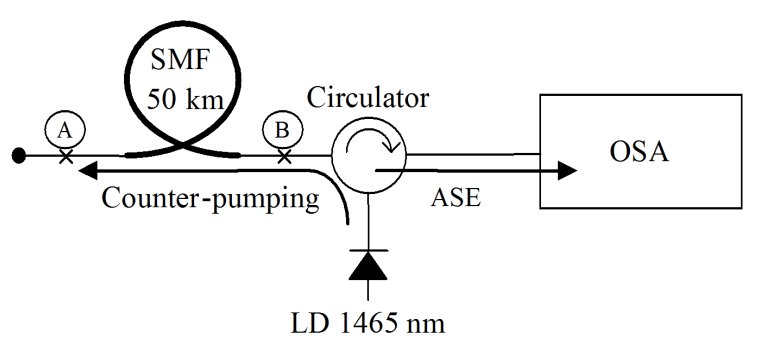

Fig.1. Experimental setup for measurement of ASE power in SMF with backward pumping.

We applied the system of coupled differential equations [8], [9] for obtaining the output signal power (at 50-km fiber end) with input power of $1 \mathrm{~mW}$ (or $0 \mathrm{dBm}$ ). Given theoretical model describes the evolution of optical wave powers, which move along the fiber: 


$$
\left\{\begin{array}{l}
\frac{\mathrm{dP}_{\mathrm{s}}(\mathrm{z})}{\mathrm{dz}}=\mathrm{g}_{\mathrm{R}}(\omega) \mathrm{P}_{\mathrm{p}} \mathrm{P}_{\mathrm{s}}-\alpha_{\mathrm{s}}(\omega) \mathrm{P}_{\mathrm{s}} \\
\frac{\mathrm{dP}_{\mathrm{p}}(\mathrm{z})}{\mathrm{dz}}=\alpha_{\mathrm{p}}(\omega) \mathrm{P}_{\mathrm{p}}
\end{array},\right.
$$

where $g_{R}(\omega)$ is a Raman gain profile in frequency domain normalized on effective fiber area $\mathrm{A}_{\mathrm{eff}} ; \mathrm{P}_{\mathrm{s}}$ and $\mathrm{P}_{\mathrm{p}}$ are signal and backward pump powers, respectively; $\alpha_{\mathrm{s}}$ and $\alpha_{\mathrm{p}}$ are attenuation coefficients for signal and pump waves, respectively; $\mathrm{z}$ is the coordinate along the fiber axis. As the signal power is small enough for $\mathrm{P}_{\mathrm{s}}<<\alpha_{\mathrm{p}} / \mathrm{g}_{\mathrm{R}}(\omega)$, then we can also ignore the pump depletion in our calculation.

\section{RESULTS AND DISCUSSION}

Obtained ASE spectra are shown in Fig.2. for $\lambda_{\mathrm{p}}=$ $1465 \mathrm{~nm}$. The general view of the spectra submitted in Fig.1. has strongly pronounced features in a qualitative interpretation of the well-known spectrum of spontaneous Raman scattering (SpRS) of light in the silica fibers. It looks like a non-uniform continuum in Stokes frequency shifts range from $0 \mathrm{THz}$ up to $27 \mathrm{THz}$.

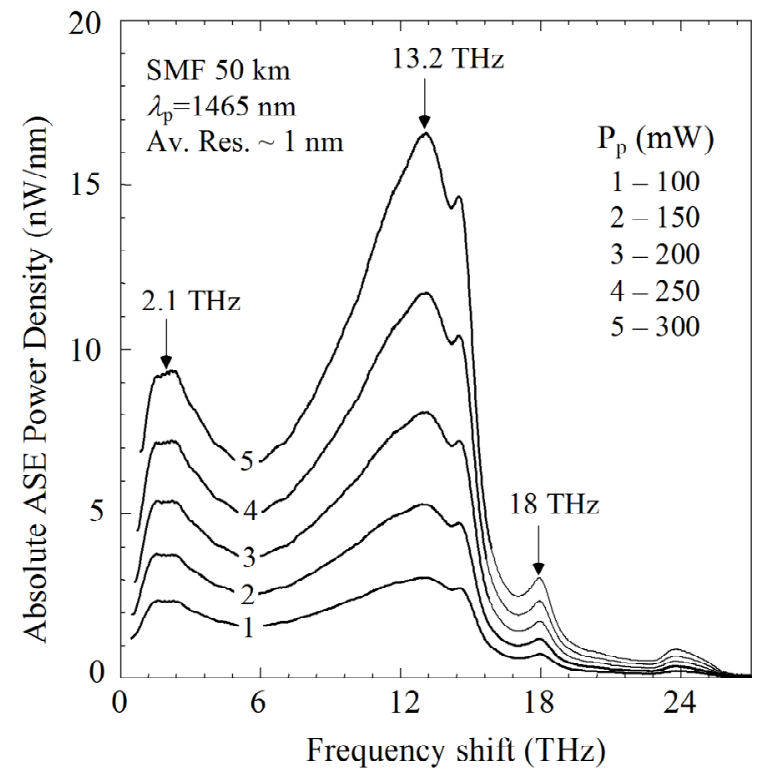

Fig.2. Absolute Stokes ASE power generated by one LD $\lambda_{p}=$ $1465 \mathrm{~nm}$ in backward pumped FRA with terahertz bandwidth.

The main difference between spontaneous Raman spectrum and stimulated Raman spectral profile is shown as the raised intensity of frequency components with small Stokes shifts approximately up to $6 \mathrm{THz}$ (see in Fig.3.). Distinction of spontaneous and stimulated Raman spectra is directly described by the quantum dynamic equation [7]. In this equation the term corresponding to spontaneous Raman scattering contains the phonon density factor $\mathrm{n}_{\mathrm{B}}(\omega)+1$, which considerably exceeds unit at $T=300 \mathrm{~K}$ in the frequency region less than $6 \mathrm{THz}$ and it infinitely grows when the frequency aspires to zero. Stimulated Raman scattering (StRS) effect forms the Raman gain profile as zero Kelvin scattering cross section, which is pointed by dotted line in Fig.3.

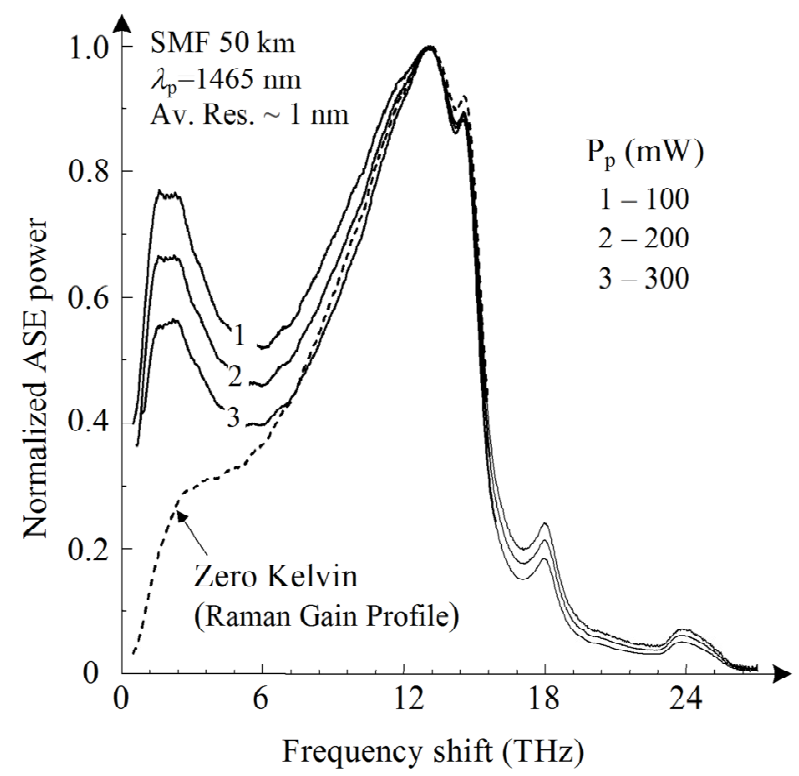

Fig.3. Normalized Stokes ASE power generated by one LD $\lambda_{p}=$ $1465 \mathrm{~nm}$ in backward pumped FRA. Normalized curves show the trend of ASE distribution to measured Raman gain profile [1] (dotted line) when the pump power is increased.

Alternatively, spontaneous Raman scattering StRS does not depend on phonon density states and it accordingly does not depend on temperature. This fact defines the difference of observable Raman gain spectrum from the measured spontaneous Raman spectrum and explains the trend of ASE distribution to Raman gain profile when the pump power increases, as one can see in Fig.3.

Another feature of spontaneous Raman scattering is a linear process by its nature and it does not depend on pump intensity. Because spontaneous Raman cross section remains constant for each studied material, the dotted lines in Fig.4. correspond to spontaneous ASE powers as function of pump power.

One can see in Fig.4. that the power gain of spontaneous optical noise in single-mode silica fiber is in the limits bounded by enough small values. Raman quantum efficiency grows no more than $\sim 45 \%$ and it corresponds to the increase of on-off Raman gain only on approximately $2.5 \mathrm{~dB}$ when the pump powers are varied 3 times, i.e. from $100 \mathrm{~mW}$ up to $300 \mathrm{~mW}$. It is possible to explain such situation from the physical point of view as follows. Rather weak ASE generation in the studied pump power range results from no coherence of the Stokes photons arising at non-elastic scattering of pump photons on huge amount of molecular phonon vibrations with different frequencies. Therefore, Stokes radiation in addition to its random phase distribution appears as distributed in a very wide frequency diapason. Both these circumstances obstruct the automatic 
establishing of the phase matching conditions necessary for coherent accumulation of Stokes radiation which would give Raman gain. In other words, Raman interaction in the core of the silica based optical fiber results in the "spreading" of pump power along the wide spectrum of Stokes frequencies.

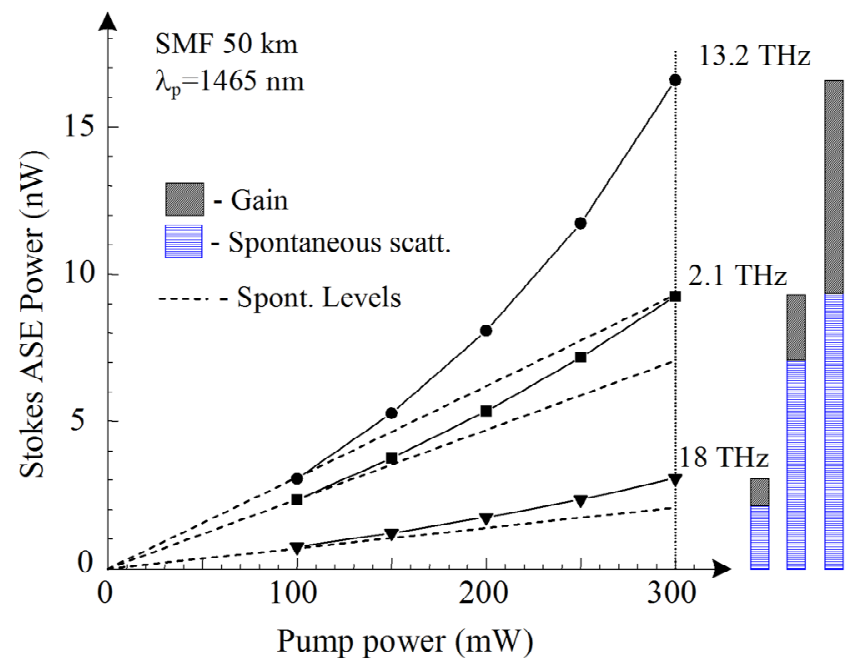

Fig.4. Stokes ASE power as function of pump power for several peaks in experimental spectra in Fig.2. (solid dots). Right hand diagram shows that the Raman gain values are only decimal parts in comparison with spontaneous scattering peaks.

The creation probability of the in-phase Stokes photons with equal frequencies inversely depends on Raman radiation bandwidth as it means that available pump power is insufficient for effective Raman noise generation. In result, spectral power distribution of ASE observable by us looks more like the spontaneous Raman scattering instead of StRS.

It should be noted that presented results are received without optical signals in studied single-mode fiber piece. At signal presence, the coherent signal power with spectral density considerably higher than the Stokes noise density starts the concurrence for possession of the pump power during the StRS process. Therefore, the ASE gain coefficients measured by us in the single-mode silica fiber have the greatest possible quantities, and noise power of real FRA cannot exceed the absolute values shown in Fig.2.

The contribution of SpRS to signal power is negligible in the case of signal amplification with counter pumping, consequently output signal power keeps the same and reaches output values almost from 78 to $93 \mu \mathrm{W}$ within wavelength range $1520-1620 \mathrm{~nm}$ or in diapason of $\mathrm{S}+\mathrm{C}+\mathrm{L}$ telecommunication window when pumping is off. It means that the ratio of signal (ASE) power from "mixture" of SpRS-StRS to signal (ASE) power just from SpRS is a parameter of the Raman on-off gain. At the presence of pumping the output signal power becomes almost twice as good owing to StRS interaction between pump and signal waves when input backward pump power equals $100 \mathrm{~mW}$. We made our calculation for investigated signal wavelength $1566 \mathrm{~nm}$, because it enters into the range of least optical losses for given type of fiber. We used experimental results for ASE also at wavelength $1566 \mathrm{~nm}$. All wavelength spectra of on-off Raman gain within total telecom $\mathrm{S}+\mathrm{C}+\mathrm{L}-$ window for signal and ASE noise are shown in Fig.5.

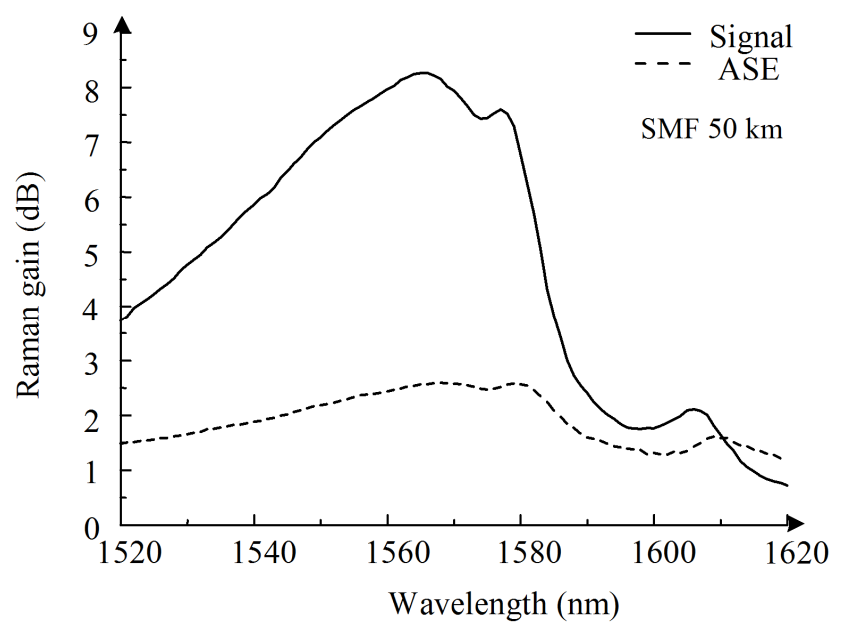

Fig.5. Calculated on-off Raman gain spectra of coherent Stokes signal (solid line) and non-coherent Stokes noise (dashed line) at pumping of $300 \mathrm{~mW}$.

So, on-off Raman gain for signal $1566 \mathrm{~nm}$ is $8.26 \mathrm{~dB}$, whereas ASE gain does not exceed $2.54 \mathrm{~dB}$ at input pump power $300 \mathrm{~mW}$. These data values are maximal in the range of $1520-1620 \mathrm{~nm}$. Efficiency of signal amplification in comparison with ASE amplification is also proved by significant OSNR. For example, OSNR at fiber output equals $45.8 \mathrm{~dB}$ with input pump power $300 \mathrm{~mW}$. The real output OSNR must be higher than the calculated one of $45.8 \mathrm{~dB}$, because ASE noise becomes maximal at signal absence and reduces at signal presence in optical fiber. Moreover, ASE noise practically does not have any effect on signal power magnitude. Thus, according to quantum limitation of noise figure [3-5] the input OSNR must be more than $91.6 \mathrm{~dB}$, which is not carried out practically. Therefore, it means that noise figure is essentially below the established limit of $3 \mathrm{~dB}$.

It is evident that the profile of on-off Raman gain spectrum repeats the shape of Raman gain efficiency coefficient $\mathrm{g}_{\mathrm{R}}(\omega)$ as much as the gain exponentially depends on input pump power, on coefficient $g_{R}(\omega)$ for the case of single signal and pump waves [8]. The Raman gain spectrum of ASE is smoother. It is caused by the equivalence of SpRS and StRS processes for the influence on output ASE power. In the case of signal amplification the influence of StRS on output signal power is several times greater than the effect of SpRS. However, the Raman gain of ASE predominates the signal Raman gain at wavelength above the value of $1610 \mathrm{~nm}$. It is explained by increasing of signal attenuation coefficient and increasing of Raman effect threshold, respectively [10]: optical losses substantially exceed signal Raman amplification at pumping of $300 \mathrm{~mW}$. In particular, output power of signal at wavelength $1620 \mathrm{~nm}$ with input backward pumping of $300 \mathrm{~mW}$ equals signal output power at $1566 \mathrm{~nm}$ when the pumping is off and it is more than 10 times lower than input signal power. 


\section{CONCLUSIONS}

Measured spectra of stochastic amplified spontaneous emission in distributed FRA with 50-km single-mode fiber in counter-pumped case are discussed in this paper. We also simulated the signal Raman amplification using numerical technique on the basis of optical wave propagation equations. Experimental and calculated data confirm that the quantum efficiency of Raman amplification of ASE does not exceed $45 \%$ and respective on-off Raman gain is no more than $2.6 \mathrm{~dB}$, whereas it is $\approx 8.3 \mathrm{~dB}$ for signal at $1566 \mathrm{~nm}$ with input power $1 \mathrm{~mW}$. The signal Raman gain is predominated over the ASE one almost everywhere inside $\mathrm{S}+\mathrm{C}+\mathrm{L}$ telecommunication window in FRA. It is shown that the backward-pumped $50-\mathrm{km}$ single-mode fiber Raman amplifier has a high OSNR performance (above $45 \mathrm{~dB}$ ) that leads to reduction of real noise figure much below the quantum limit.

\section{ACKNOWLEDGMENT}

We are indebted to Dr. P. Korotkov, who encouraged us to write this paper and for discussions of optical noise that have improved our understanding of this subject.

\section{REFERENCES}

[1] Islam, M.N. (2002). Raman amplifiers for telecommunications. IEEE Journal of Selected Topics in Quantum Electronics, 8 (3), 548-559.

[2] Kidorf, H., Rottwitt, K., Nissov, M., Ma, M., Rabarijaona, E. (1999). Pump interactions in $100 \mathrm{~nm}$ bandwidth Raman amplifier. IEEE Photonics Technology Letters, 11 (5), 530-532.

[3] Yamamoto, Y., Inoue, K. (2003). Noise in amplifiers. Journal of Lightwave Technology, 21 (11), 2895-2915.

[4] Haus, H.A. (2000). Noise figure definition valid from RF to optical frequancies. IEEE Journal of Selected Topics in Quantum Electronics, 6 (2), 240-247.

[5] Henry, C.H., Kazarinov, R.F. (1996). Quantum noise in photonics. Reviews of Modern Physics, 68 (3), 801853.

[6] Agrawal, G.P. (2002). Fiber-Optic Communication Systems (3th ed.). John Wiley \& Sons.

[7] Felinskyi, G.S., Korotkov, P.A. (2006). Simulation of multiwavepumped fiber Raman amplifiers. Semiconductor Physics, Quantum Electronics \& Optoelectronics, 9 (3), 83-93.

[8] Bromage, J. (2004). Raman amplification for fiber communications systems. Journal of Lightwave Technology, 22 (1), 79-93.

[9] Namiki, S., Emori, Y. (2001). Ultrabroad-band Raman amplifiers pumped and gain-equalized by wavelengthdivision-multiplexed high-power laser diodes. IEEE Journal of Selected Topics in Quantum Electronics, 7 (1), 3-16.

[10] Dyriv, M.Y., Felinskyi, G.S., Korotkov, P.A. (2013). Fiber attenuation irregularities and simulation of Raman amplification band. In Conference on Laser and Fiber-Optical Network Modeling, 11-13 September 2013, Sudak, Crimea, Ukraine, 44-46.

Received July 11, 2014. Accepted June 18, 2015. 